\title{
Validation of putative reference genes for gene expression studies in human hepatocellular carcinoma using real-time quantitative RT-PCR
}

\author{
Vito R Cicinnati ${ }^{\dagger 1,2}$, Qingli Shen ${ }^{\dagger 1}$, Georgios C Sotiropoulos ${ }^{2}$, \\ Arnold Radtke ${ }^{2}$, Guido Gerken ${ }^{1}$ and Susanne Beckebaum*1,2
}

\begin{abstract}
Address: ${ }^{1}$ Department of Gastroenterology and Hepatology, University Hospital Essen, University of Duisburg-Essen, Essen, Germany and ${ }^{2}$ Department of General, Visceral and Transplantation Surgery, University Hospital Essen, University of Duisburg-Essen, Essen, Germany

Email: Vito R Cicinnati - vito.cicinnati@uni-due.de; Qingli Shen - qingli.shen@uni-due.de; Georgios C Sotiropoulos - gsotirop@yahoo.com; Arnold Radtke - arnold.radtke@uni-due.de; Guido Gerken - guido.gerken@uk-essen.de; Susanne Beckebaum* - susanne.beckebaum@unidue.de

* Corresponding author †Equal contributors
\end{abstract}

Published: 27 November 2008

BMC Cancer 2008, 8:350 doi:10.1 |86/147|-2407-8-350
Received: 27 March 2008

Accepted: 27 November 2008

This article is available from: http://www.biomedcentral.com/I47I-2407/8/350

(c) 2008 Cicinnati et al; licensee BioMed Central Ltd.

This is an Open Access article distributed under the terms of the Creative Commons Attribution License (http://creativecommons.org/licenses/by/2.0), which permits unrestricted use, distribution, and reproduction in any medium, provided the original work is properly cited.

\begin{abstract}
Background: Reference genes, which are often referred to as housekeeping genes are frequently used to normalize mRNA levels between different samples in quantitative reverse transcription polymerase chain reaction (qRT-PCR). The selection of reference genes is critical for gene expression studies because the expression of these genes may vary among tissues or cells and may change under certain circumstances. Here, a systematic evaluation of six putative reference genes for gene expression studies in human hepatocellular carcinoma (HCC) is presented.
\end{abstract}

Methods: Six genes, beta-2-microglobulin (B2M), glyceraldehyde-3-phosphate dehydrogenase (GAPDH), hydroxymethyl-bilane synthase (HMBS), hypoxanthine phosphoribosyl-transferase I (HPRTI), succinate dehydrogenase complex, subunit $A(S D H A)$ and ubiquitin $C$ (UBC), with distinct functional characteristics and expression patterns were evaluated by qRT-PCR. Inhibitory substances in RNA samples were quantitatively assessed and controlled using an external RNA control. The stability of selected reference genes was analyzed using both geNorm and NormFinder software.

Results: HMBS and GAPDH were identified as the optimal reference genes for normalizing gene expression data between paired tumoral and adjacent non-tumoral tissues derived from patients with HCC. HMBS, GAPDH and $U B C$ were identified to be suitable for the normalization of gene expression data among tumor tissues; whereas the combination of HMBS, B2M, SDHA and GAPDH was suitable for normalizing gene expression data among five liver cancer cell lines, namely Hep3B, HepG2, HuH7, SK-HEP-I and SNU-I82. The determined gene stability was increased after exclusion of RNA samples containing relatively higher inhibitory substances.

Conclusion: Of six genes studied, HMBS was found to be the single best reference gene for gene expression studies in HCC. The appropriate choice of combination of more than one reference gene to improve qRT-PCR accuracy depends on the kind of liver tissues or cells under investigation. Quantitative assessment and control of qRT-PCR inhibitors using an external RNA control can reduce the variation of qRT-PCR assay and facilitate the evaluation of gene stability. Our results may facilitate the choice of reference genes for expression studies in HCC. 


\section{Background}

Real-time quantitative reverse transcription (qRT)polymerase chain reaction (PCR) is a rapid, sensitive and reliable method for gene expression studies. It is inherently an indirect method of measurement, and variabilities exist in the various steps of the GRT-PCR which may lead to severe misinterpretation of the results. The latter may be due to different amounts and quality of starting material, variable enzymatic efficiencies (i.e. efficiency of retrotranscription from RNA to complementary DNA (cDNA), and PCR efficiency) between samples and runs, operator errors, and differences between tissues or cells in overall transcriptional activity $[1,2]$. Thus, proper normalization strategy is necessary for reliable quantitative information to be extracted from this variable system.

Various strategies have been explored in an attempt to normalize these variations, and it is generally accepted that gene-expression levels should be normalized by carefully selected and stably expressed reference genes [2-4]. The success of this procedure is highly dependent on the choice of the appropriate reference genes. An ideal reference gene should be unaffected by the experimental conditions and should have low variation in gene expression. Otherwise, the detection of small changes becomes unfeasible, producing results that may be entirely incorrect [5]. Several reports have provided the evidence that the expression of most commonly used reference genes varies among tissues or cells and may also change under certain environmental circumstances [6-11]. For instance, a recent report indicated the de-regulation of common reference genes in hepatocellular carcinoma (HCC) arising from hepatitis $\mathrm{C}$ virus (HCV) infected liver [12]. Therefore, it is critical to perform preliminary evaluation studies, aimed at identifying the most stably expressed reference genes in individual tissues and distinct circumstances for each single experiment.

HCC is the sixth most frequently diagnosed cancer and the third most common cause of cancer mortality in the world [13], but the molecular mechanisms of hepatocarcinogenesis, including gene expression variation, are not well understood. Therefore, the number of studies assessing global gene expression profiles of HCC has increased exponentially in recent years [14-20], and the identification of optimal reference genes is necessary for correct gene expression profiling of HCC. In the current study, we validated the stability of six putative reference genes in liver cancer cell lines, tumoral tissues and adjacent nontumoral tissues from 20 HCC patients. Two algorithms based on different strategies, geNorm and NormFinder, were used for data analysis.

\section{Methods \\ Primer design}

Primers for RT-PCR assays of $H M B S$ and $U B C$ were designed using Primer Express v2.0 (Applied Biosystems, Foster City, California, USA). Primer sequences for B2M, GAPDH, HPRT1 and SDHA were obtained from the realtime PCR primer and probe database (RTPrimerDB) [21]. Special attention was given to primer length, annealing temperature, base composition and 3'-end stability. To ensure optimal DNA polymerization efficiency, amplicon length ranged between 69 and $113 \mathrm{bp}$. Exon and intron boundaries were determined aligning primers with corresponding human genomic sequences downloaded from GenBank http://www.ncbi.nlm.nih.gov/Genbank. RTPCR primers for each gene were located on different exons or directly spanning exon-exon boundaries of the genomic sequence to minimize amplification from any contaminating genomic DNA that may remain following DNase treatment. To ensure that gene transcripts used as standards display a similar secondary structure with native RNA, primers for cloning were designed to have binding sites located at least 20 nucleotides external to the binding sites of primers for assays. The sequences of primers for cloning and the length of amplicons are shown in Supplementary table 1 in additional file 1 .

\section{Samples}

Human liver cancer cell lines Hep3B, HepG2, SK-HEP-1 and SNU-182 were obtained from the American Type Culture Collection (ATCC, Manassas, USA). Human HCC cell line $\mathrm{HuH} 7$ was a kind gift from Dr. Brigitte Pützer. Нер3B, HepG2, HUH7 and SK-HEP-1 were grown in DMEM medium (high glucose, with glutamine and sodium pyruvate) supplemented with $10 \%(\mathrm{v} / \mathrm{v})$ heat-inactivated fetal bovine serum (FBS), $100 \mathrm{U} / \mathrm{ml}$ penicillin, $100 \mu \mathrm{g} / \mathrm{ml}$ streptomycin (all from PAA Laboratories Pasching, Austria), and $0.1 \mathrm{mM} \mathrm{GIBCO}{ }^{\mathrm{TM}} \mathrm{MEM}$ Non Essential Amino Acids (Invitrogen Corporation, Carlsbad, CA). SNU-182 was grown in RPMI 1640 medium containing $1 \mathrm{mM}$ sodium pyruvate, $2 \mathrm{mM}$ L-glutamine, $4500 \mathrm{mg} / \mathrm{L}$ glucose, $10 \mathrm{mM}$ HEPES, and $1500 \mathrm{mg} / \mathrm{L}$ sodium bicarbonate, supplemented with $10 \%(\mathrm{v} / \mathrm{v})$ heat-inactivated FBS, $100 \mathrm{U} /$ $\mathrm{ml}$ penicillin and $100 \mu \mathrm{g} / \mathrm{ml}$ streptomycin (all from PAA Laboratories). Stock cultures were maintained at $37^{\circ} \mathrm{C}$ in a humidified, $5 \% \mathrm{CO}_{2}$ incubator and were routinely passaged at 3 to 4 day intervals. Tumoral and non-tumoral liver tissues were obtained from 20 patients with HCC undergoing liver resection. Most of those were paired tumoral and adjacent non-tumoral tissues. All tissue samples were snap frozen in liquid nitrogen and stored till use at $-80^{\circ} \mathrm{C}$. The study was approved by the local ethics committee and all patients provided written informed consent. Detailed information regarding cell lines, patients and tissue samples is shown in Supplementary tables 2 and 3 in additional file 1. 


\section{Total RNA isolation and characterization}

Total RNA was extracted using the miRNeasy Mini Kit (Qiagen, Hilden, Germany), according to the manufacturer's instructions. Genomic DNA was eliminated by a DNase-on-column treatment with RNase-free DNase set (Qiagen). The purity of extracted RNA was assessed by measuring the optical density (OD) at wavelengths of 230 $\mathrm{nm}, 260 \mathrm{~nm}$ and $280 \mathrm{~nm}$ using a quartz cuvette with Eppendorf Biophotometer (Eppendorf, Hamburg, Germany). Absorbance ratio at 260/280 nm and 260/230 nm was used to assess the purity of the RNA samples. The integrity and quantity of extracted RNA were assessed by evaluating the capillary electrophoresis trace with Agilent 2100 bioanalyzer (Agilent Technologies, Waldbronn, Germany). The integrity of the samples was determined by RNA integrity number (RIN) algorithm, which assigns a score from 10 to 1 , where level 10 represents a completely intact RNA, and level 1 represents a highly degraded RNA [22]. Total RNA from cell lines was extracted at the exponential growing phase. Liver tissue samples were disrupted and homogenized in QIAzol lysis reagent with TissueRuptor (Qiagen) for total RNA isolation.

\section{Construction of absolute standard curves}

In vitro transcripts of each gene were generated as standards. RT-PCR products of each gene were purified with Wizard ${ }^{\circledR}$ SV Gel and PCR Clean-Up System (Promega Corporation, Madison, WI, USA), and then cloned with PGEM $^{\circledR}$-T easy vector system (Promega), according to the manufacturer's instructions. Plasmid DNA was isolated and purified with Wizard ${ }^{\circledR}$ plus SV Minipreps DNA purification system (Promega). The orientation and sequence of the inserts were confirmed by restriction endonuclease digestion with EcoRI (Roche Molecular Biochemicals, Mannheim, Germany) followed by agarose gel electrophoresis with ethidium bromide staining, and gene sequencing with BigDye ${ }^{\circledast}$ Terminator v1.1 Cycle Sequencing Kit (Applied Biosystems, Foster City, CA, USA) using the T7 Promoter Primer (Promega). Bacterial clones with correct inserts were cultured again for isolation and purification of plasmid DNA with PureYield ${ }^{\mathrm{TM}}$ Plasmid Midiprep System (Promega). Plasmid DNA was linearized with restriction endonuclease SaI I (Roche) or NcoI (Promega) and transcribed into RNA with MEGAscript ${ }^{\circledR}$ T7 or Sp6 kit (Ambion, Austin, TX, USA). Transcripts were purified with MEGAclear ${ }^{\mathrm{TM}}$ kit (Ambion) and plasmid DNA was eliminated by DNase treatment before and after the purification of the transcripts with TURBO DNase I (Ambion) and TURBO DNA-free ${ }^{\mathrm{TM}}$ kit (Ambion) respectively. DNase treated samples were desalted with DNase inactivation reagent included in TURBO DNA-free ${ }^{\mathrm{TM}}$ kit. The quantity and quality of the transcripts were evaluated with Eppendorf Biophotometer (Eppendorf), denatured agarose gel electrophoresis and Agilent 2100 bioanalyzer
(Agilent). Five points of 10 -fold serial dilution of each transcript were used to build the standard curve for each gene. The copy number range of each standard curve was set according to the approximate expression level of the individual gene determined by preliminary experiments and encompassed the copy number range of the genes in the samples. The copy numbers of the standards are shown in Supplementary table 4 in additional file 1.

\section{qRT-PCR inhibitor detection}

qRT-PCR inhibitor was detected in each sample and standard using Alien ${ }^{\circledR}$ QRT-PCR Inhibitor Alert (Stratagene, La Lolla, CA, USA), according to the manufacturer's instructions. Briefly, each sample was spiked with $10^{5}$ copies of alien RNA transcript, an in-vitro transcribed RNA being non-homologous to the sequences currently available in GenBank. Increases in the threshold cycle $(\mathrm{Ct})$ value for amplification of alien RNA in samples were compared with alien RNA alone and calculated to determine the presence of inhibitory substances in the samples [23].

\section{Real-time quantitative RT-PCR}

Real-time quantitative RT-PCR (qRT-PCR) was performed with the SYBR Green QuantiTect RT-PCR Kit (Qiagen), according to the manufacturer's instructions. For each sample, 20 ng total RNA was used in the assay and all the genes were tested with the same panel of RNA samples. RT negative reactions were performed for standards and sample RNA. All standards and samples were run in duplicate on 96-well reaction plates with the $\mathrm{iQ}^{\mathrm{TM}} 5$ Multicolor RealTime PCR Detection System (Bio-Rad, Hercules, CA, USA). Reactions were prepared in a total volume of $25 \mu \mathrm{l}$ containing $20 \mathrm{ng}$ RNA in $5 \mu$ l volume, $1.25 \mu \mathrm{l}$ of each 10 $\mu \mathrm{M}$ primer (500 $\mathrm{nM}), 12.5 \mu$ of SYBR Green master mix, $0.25 \mu \mathrm{l}$ of RT mix and $4.75 \mu \mathrm{l}$ RNase/DNase-free sterile water. No template controls were run for each master mix and primer pair. After performing preliminary gradient real-time RT-PCR assays, the optimal annealing temperature for all the primer pairs resulted to be $56.4^{\circ} \mathrm{C}$. At this temperature the lowest $\mathrm{Ct}$ value was generated as well as a sharp melting peak, with no amplification of non-specific products or primer-dimer artifacts (see Additional file 2, $3,4,5,6,7)$. The conditions of reverse transcription and primer concentrations were set according to the recommendations in the manufacturer's instructions and confirmed by preliminary experiments which generated correlation coefficient $\left(\mathrm{R}^{2}\right)$ of each standard curve above 0.99. The cycle conditions were as follows: reverse transcription at $50^{\circ} \mathrm{C}$ for $30 \mathrm{~min}$, DNA polymerase activation and RT enzyme inactivation at $95^{\circ} \mathrm{C}$ for $15 \mathrm{~min}$, followed by 40 cycles of denaturation at $94^{\circ} \mathrm{C}$ for $15 \mathrm{~s}$, primer annealing at $56.4^{\circ} \mathrm{C}$ for $30 \mathrm{~s}$, elongation at $72^{\circ} \mathrm{C}$ for $30 \mathrm{~s}$. The data collection step was performed at a temperature that was $3^{\circ} \mathrm{C}$ lower than the melting temperature of each amplicon and higher than that of primer-dimers of each 
primer pair. This cycle was followed by a melting curve analysis, ranging from $55^{\circ} \mathrm{C}$ to $95^{\circ} \mathrm{C}$, with temperature increasing steps of $0.5^{\circ} \mathrm{C}$ every $10 \mathrm{~s}$. Baseline and threshold values were automatically determined for all plates using the Bio-Rad iQ5 Software 2.0. The obtained data were analyzed using geNorm (version 3.5, written by Vandesompele J. et al) and NormFinder software (written by Andersen C.L. et al).

\section{Results and discussion}

Six putative reference genes from different abundance and functional classes were selected for evaluation: $B 2 \mathrm{M}$, GAPDH, HMBS, HPRT1, SDHA, and UBC (Table 1). Ribosome RNAs, like 28s rRNA and 18s rRNA were not included in this study, because imbalance between rRNA and mRNA fractions has been observed and rRNA transcription is affected by biological factors and drugs [2427]. Gene sequence information deposited in GenBank under the Accession Numbers was used to design RT-PCR primers (Table 2). The qRT-PCR efficiency for each single primer pair was determined in real-time RT-PCR using serial 10-fold dilutions of in vitro transcripts of each gene. Expression patterns of the six reference genes have been subsequently evaluated in different sample groups.

\section{RNA quantity and quality determination}

All RNA samples were examined for their purity, integrity and concentration. The absorbance ratio at 260/280 nm of all the samples were all over 2.0, except for one sample with a ratio of 1.85 , indicating all the samples were free from proteins potentially accumulating during the RNA extraction procedure. The absorbance ratios at 260/230 $\mathrm{nm}$ were $0.43 \sim 2.23$, indicating possible contamination of some samples with carbohydrates, peptides, phenols or aromatic compounds [28]. Although samples with a low OD 260/230 ratio may interfere with downstream process like RT-PCR [28], only a few studies using RT-PCR methods have taken the OD 260/230 ratio into consideration when evaluating RNA sample purity. In addition, there is no generally accepted cutoff value of OD 260/230 ratio that allows researchers to determine the suitability of a sample to be used in qRT-PCR. Thus, RNA samples were additionally subjected to qRT-PCR inhibitor detection for the mining of the relationship between OD 260/230 ratio and qRT-PCR inhibition. All RNA samples were determined to be intact or to have mild loss of integrity (RIN > 6.5) using the RIN algorithm [22]. A RIN higher than 5 was recommended as good total RNA quality and a RIN higher than 8 as perfect total RNA quality for downstream qRT-PCR application [29]. Therefore, the integrity of all RNA samples was suitable for the use in our experiments. The absorbance ratio at 260/280 nm, 260/230 nm and RIN are shown in Supplementary table 5 in additional file 1.

\section{Presence of qRT-PCR inhibitors in purified RNA samples} Increases of the $\mathrm{Ct}$ value $(\Delta \mathrm{Ct})$, for amplification of alien RNA in 100 ng samples compared with alien RNA alone ranged between $0.16 \sim 2.37$. When the samples were diluted 5-fold, increases of the Ct value, ranged between $0.14 \sim 0.92$, indicating that the inhibition of qRT-PCR by inhibitors was significantly reduced by simply diluting the experimental RNA samples (Figure 1), but was not reduced to an acceptable level in some samples.

Pearson's correlation analysis between the OD 260/230 ratios and $\Delta \mathrm{Ct}$ values resulted in a Pearson's $\mathrm{r}$ of -0.2420 , $\mathrm{r}^{2}$ of 0.0586 , and a value of 0.175 , indicating no statistically significant linear relationship between the two variables (Table 3). Data shown here suggest that an alien RNA control, but not the OD 260/230 ratio, is an direct and specific way for the detection of qRT-PCR inhibitors

Table I: Putative reference genes evaluated.

\begin{tabular}{|c|c|c|c|c|c|c|}
\hline Gene & $\begin{array}{l}\text { GenBank accession } \\
\text { number }\end{array}$ & Name & Function & Genomic localization & Gene Aliases & IMAGE \\
\hline$B 2 M$ & NM 004048 & Beta-2-microglobulin & $\begin{array}{l}\text { Beta-chain of major } \\
\text { histocompatibility } \\
\text { complex } \\
\text { class I molecules }\end{array}$ & $15 q 2 \mid-q 22$ & & 51940 \\
\hline GAPDH & NM 002046 & $\begin{array}{l}\text { Glyceraldehyde-3- } \\
\text { phosphate } \\
\text { dehydrogenase }\end{array}$ & $\begin{array}{l}\text { Oxidoreductase in } \\
\text { glycolysis and } \\
\text { gluconeogenesis }\end{array}$ & $12 p \mid 3$ & G3PD & 510510 \\
\hline HMBS & NM 000190.3 & $\begin{array}{l}\text { Hydroxymethyl-bilane } \\
\text { synthase }\end{array}$ & $\begin{array}{l}\text { Heme synthesis, } \\
\text { porphyrin metabolism }\end{array}$ & IIq23 & & 245564 \\
\hline HPRTI & NM 000194.1 & $\begin{array}{l}\text { Hypoxanthine } \\
\text { phosphoribosyl- } \\
\text { transferase I }\end{array}$ & $\begin{array}{l}\text { Purine synthesis in } \\
\text { salvage pathway }\end{array}$ & $X q 26$ & HGPRT & 345845 \\
\hline SDHA & NM 004168.2 & $\begin{array}{l}\text { Succinate dehydrogenase } \\
\text { complex, subunit A }\end{array}$ & $\begin{array}{l}\text { Electron transporter in } \\
\text { the TCA cycle and } \\
\text { respiratory chain }\end{array}$ & $5 p 15$ & FP; SDH2; SDHF & 375812 \\
\hline$U B C$ & NM 021009.3 & Ubiquitin C & Protein degradation & $12 q 24$ & & 510582 \\
\hline
\end{tabular}


Table 2: Details of primers and amplicons for the 6 evaluated genes.

\begin{tabular}{|c|c|c|c|c|c|c|}
\hline Gene & Primer & Sequence $\left(5^{\prime} \rightarrow 3^{\prime}\right)$ & Genomic Position & Amplicon Length & $\begin{array}{l}\text { correlation coeffi- } \\
\text { cients }\left(R^{2}\right)\end{array}$ & $\begin{array}{c}\text { qRT-PCR Efficiency } \\
(\%)\end{array}$ \\
\hline \multirow[t]{2}{*}{$B 2 M$} & Forward & $\begin{array}{l}\text { CTCCGTGGCCTTAGC } \\
\text { TGTG }\end{array}$ & Ist Exon & $69 \mathrm{bp}$ & 0.998 & 90.1 \\
\hline & Reverse & $\begin{array}{l}\text { TTTGGAGTACGCTGG } \\
\text { ATAGCCT }\end{array}$ & Ist/2nd exon & & & \\
\hline \multirow[t]{2}{*}{ GAPDH } & Forward & $\begin{array}{l}\text { TGCACCACCAACTGC } \\
\text { TTAGC }\end{array}$ & 7th Exon & 87 bp & 0.997 & 92 \\
\hline & Reverse & $\begin{array}{l}\text { GGCATGGACTGTGGT } \\
\text { CATGAG }\end{array}$ & 7th/8th exon & & & \\
\hline \multirow[t]{2}{*}{ HMBS } & Forward & $\begin{array}{l}\text { TGCAACGGCGGAAGA } \\
\text { AAA }\end{array}$ & Ist/2nd Exon & 113 bp & 0.998 & 91.0 \\
\hline & Reverse & $\begin{array}{l}\text { ACGAGGCTTTCAATG } \\
\text { TTGCC }\end{array}$ & $3 r d$ exon & & & \\
\hline \multirow[t]{2}{*}{ HPRTI } & Forward & $\begin{array}{l}\text { TGACACTGGCAAAAC } \\
\text { AATGCA }\end{array}$ & 6th Exon & $94 \mathrm{bp}$ & 0.997 & 94.9 \\
\hline & Reverse & $\begin{array}{l}\text { GGTCCTTTTCACCAG } \\
\text { CAAGCT }\end{array}$ & 6th/7th exon & & & \\
\hline \multirow[t]{2}{*}{ SDHA } & Forward & $\begin{array}{l}\text { TGGGAACAAGAGGGC } \\
\text { ATCTG }\end{array}$ & 2nd Exon & 86 bp & 0.996 & 87.8 \\
\hline & Reverse & $\begin{array}{l}\text { CCACCACTGCATCAA } \\
\text { ATTCATG }\end{array}$ & $3 r d$ exon & & & \\
\hline \multirow[t]{2}{*}{$U B C$} & Forward & $\begin{array}{l}\text { CGGTGAACGCCGATG } \\
\text { ATTAT }\end{array}$ & Ist Exon & 124 bp & 0.994 & 88.4 \\
\hline & Reverse & $\begin{array}{l}\text { ATCTGCATTGTCAAG } \\
\text { TGACGA }\end{array}$ & Ist/2nd exon & & & \\
\hline
\end{tabular}

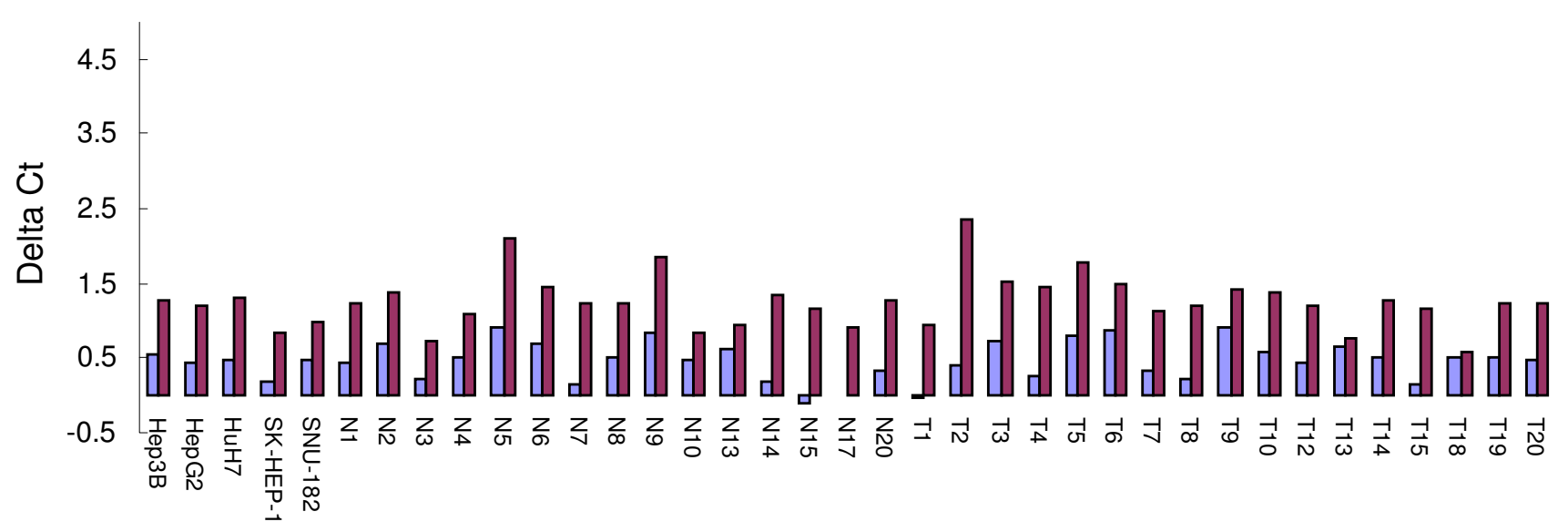

\section{Samples}

\section{$\square$ 20ng RNA $\square$ 100ng RNA}

\section{Figure I}

QRT-PCR inhibitor detection in all samples. $\triangle \mathrm{Ct}$ for amplification of alien RNA in samples of $100 \mathrm{ng}$ and $20 \mathrm{ng}$ RNA respectively were compared with that of Alien RNA alone. Each sample was spiked with $10^{5}$ copies of alien RNA transcripts. 
Table 3: Pearson's correlation analysis between the existence of qRT-PCR inhibitors and OD 260/230.

\begin{tabular}{ccccccc}
\hline & median & 75th & Mean & STD & Normality test & Pearson's correlation coefficient \\
\hline$\Delta \mathrm{Ct}^{*}$ & 0,49 & 0.675 & 0.47 & 0.26 & $\mathrm{P} \geq 0.20$ & $\mathrm{r}=-0.2420\left(\mathrm{r}^{2}=0.0586\right), \mathrm{P}=0.175$ \\
$\mathrm{OD} 260 / 230$ & 1.49 & 1.965 & 1.49 & 0.48 & $\mathrm{P} \geq 0.15$ & \\
\hline
\end{tabular}

Both of the variables were determined to be approximately normally distributed prior to the calculation of Pearson's correlation coefficient. * Amplification of alien RNA in $20 \mathrm{ng}$ RNA samples compared with alien RNA alone; OD260/230: the ratio of optical density (OD) at wavelength of $260 \mathrm{~nm}$ to that at $230 \mathrm{~nm}$. 75th: 75 th percentile.

in RNA samples. Thus, the selection of samples in this study was based on the quantitative assessment of qRTPCR inhibitors using an alien control RNA. Samples with a $\Delta \mathrm{Ct}$ value higher than the 75 th percentile of all the samples, namely 0.675 , were excluded from the analyses for the determination of suitable reference genes. For paired tissues, both tissues were excluded from the analysis if the $\Delta \mathrm{Ct}$ value of one of them exceeded the cutoff value.

There is a variety of inhibitors potentially affecting the efficiency of qRT-PCR reactions which may be co-purified with RNA samples, depending on the source of starting material and the methods of extraction. Tissues are among the common sample types known to contain inhibitors like collagen [30]. Inhibitors from experimental reagents include phenol, ethanol, guanidine, EDTA and others [23]. Thus, it is important to assess the existence of qRTPCR inhibitors in RNA samples from tissues prior to the qRT-PCR assay.

We found no significant correlation between the OD 260/ 230 ratio and the level of qRT-PCR inhibitors in RNA samples. Moreover, it has been shown that some inhibitors may directly bind to nucleotides [31]. Therefore, whether a modification of the RNA extraction procedure or a repurification of RNA samples will reduce the level of qRT-PCR inhibitors needs further validation.

\section{Presence of contaminating DNA in standards and purified RNA samples}

RT negative reactions for standards confirmed completely removal of plasmid DNA. There were PCR amplifications in RT negative reactions of B2M and GAPDH in two samples with Ct values much lower than those of RT positive reactions of the same samples, indicating a negligible copy number of amplified contaminating DNA (see additional file 8). There were no PCR amplifications of the other four genes in RT negative reactions for the samples.

\section{Standard curves and qRT-PCR efficiency of each primer pair}

The qRT-PCR efficiency of each primer pair was determined by serial dilutions of homologous external RNA of each gene. Homologous RNA standards are most suitable for two reasons [32]: (i) RNA standards (as opposed to DNA) must be used to control the variability during the RT step; (ii) homologous external RNA standards share the same primer binding site as the native RNA and has the same intervening sequence, and hence are most likely to have the same or very similar RT and PCR efficiencies. DNA standards, such as plasmid DNA and PCR fragments are generally used, assuming that the RT variability is negligible, because the subsequent PCR assays are generally used to calculate PCR efficiency. The use of DNA standards can be an accurate estimation of qRT-PCR efficiency only when there is minimal RT variability. It is worth noting that in vitro transcribed RNA from PCR products is not the same as full length mRNA, concerning secondary or tertiary mRNA structures which may affect RT efficiency. Thus, for in vitro transcribed RNA, it is important to have sequences upstream and downstream from the amplified sequence identical or similar to the "natural" target. The use of one-step qRT-PCR in this study further reduced the variability of qRT-PCR by lowering the contamination risk because of circumventing the need to open the reaction tubes between RT and PCR steps. To adapt the background of the standards to the native RNA samples, each dilution of the standards was spiked with $20 \mathrm{ng}$ E. coli total RNA consisting of the same amount of nucleic acids as the native RNA samples.

Efficiency of qRT-PCR (E) of primer pairs ranged from $87.8 \%$ to $94.9 \%$, and correlation coefficients $\left(\mathrm{R}^{2}\right)$ ranged from 0.994 to 0.998 (Table 2). The amplification of alien RNA transcripts among standards and 20 ng native RNA samples was at a similar level, thus indicating analogical qRT-PCR efficiency (Figure 2).

\section{Expression levels of candidate reference genes}

According to the average copy number of each gene in 20 ng purified total RNA of each sample, the six tested genes belong to various abundance classes, with an approximately 10,000-fold expression difference between the most abundant $(B 2 M)$ and the rarest $(U B C)$ transcript. The expression levels for individual genes were also different among different sample panels. Four out of six genes showed an expression pattern in cell lines $>$ tumoral tissues $>$ non-tumoral tissues, SDHA showed an expression 


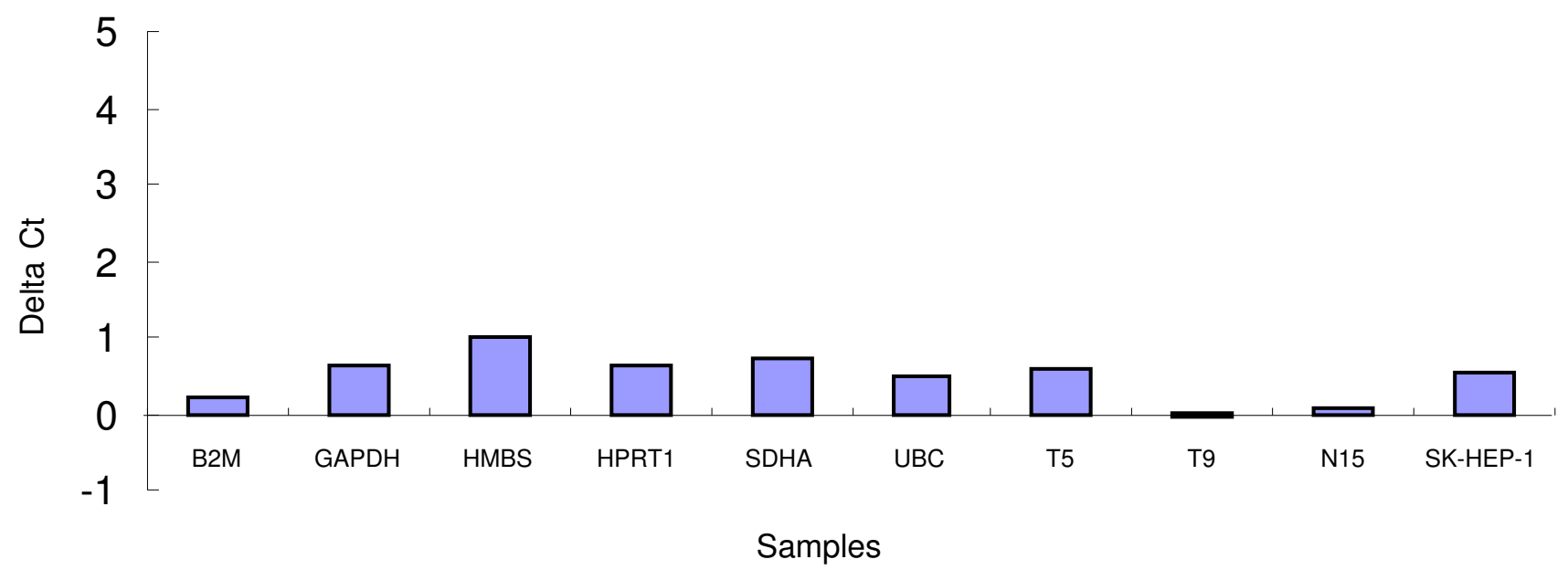

\section{Figure 2}

QRT-PCR inhibitor detection in standards and several RNA samples. $\Delta$ Ct for amplification of alien RNA in standards spiked with $20 \mathrm{ng}$ E. coli total RNA and $20 \mathrm{ng}$ RNA from several RNA samples were compared with that of alien RNA alone. The tissue samples contained relatively high and low abundance of qRT-PCR inhibitor existence as determined by the qRTPCR detection among all samples. Each sample was spiked with $10^{5}$ copies of alien RNA transcripts.

pattern in cell lines $>$ non-tumoral tissues $\approx$ tumoral tissues, and B2M showed an expression pattern in tumoral tissues > non-tumoral tissues > cell lines (Figure 3).

\section{Expression stability of putative reference genes}

The data obtained for each sample and each reference gene were analyzed using both geNorm and NormFinder $[1,33]$. GeNorm provides a ranking of the tested genes based on the reference gene stability measure $M$ which is defined as the average pair-wise variation of a particular gene compared with all other control genes. Genes with higher $M$ values have greater variations of expression. Additionally, the assessment of the pair-wise variations $\left(\mathrm{V}_{\mathrm{n} / \mathrm{n}+1}\right)$ between each combination of sequential normalization factors allows the identification of the optimal number of reference genes. NormFinder, whose strategy is rooted in a mathematical model of gene expression, provides a ranking of the tested genes based on a direct measure of both overall expression variation and the variation between sample subgroups of candidate reference genes. The stability of genes was shown as stability value, and genes with lower stability values have higher expression stability. NormFinder has been reported to show relatively low sensitivity towards co-regulation of the candidate normalization genes [33].

The analysis by geNorm suggested that all studied genes reached a high expression stability with low $M$ values (Table 4 and 5), below the default limit of $M=1.5[1]$. The optimal reference genes for normalizing gene expression data between paired tumoral and non-tumoral liver tissues were HMBS and GAPDH (Table 4; Figure 4). HMBS, $G A P D H$ and $U B C$ were identified to be suitable for the normalization of gene expression data among tumor tissues. The combination of HMBS, B2M, SDHA and $G A P D H$ was suitable for normalizing gene expression data among five liver cancer cell lines, yielding a $\mathrm{V}_{4 / 5}$ value of 0.175 , which is close to the cutoff value 0.15 . The reason why there was no low enough $\mathrm{V}$ value in cell lines may be due to the limited number of cell lines or candidate reference genes included in the evaluation.

The results of the analysis by NormFinder appeared to be identical to the one determined using geNorm in each sample panel, except for the position of two genes with close stability, GAPDH and SDHA, whose ranking was inverted in paired tissues (Table 4). Interestingly, the stable value of the best combination of two genes determined by NormFinder for paired tissues was slightly higher than that of HMBS alone (Table 4), indicating HMBS alone may be sufficient as reference gene for expression studies in paired tissues.

When comparing the data of the paired tissue group in which 10 tissue RNA samples with high inhibitory substances were excluded, a higher stability of each gene, and a higher similarity between the two algorithms became evident (Table 4; Figure 4), indicating that the existence of qRT-PCR inhibitors in RNA samples can increase the variation of qRT-PCR assay. 


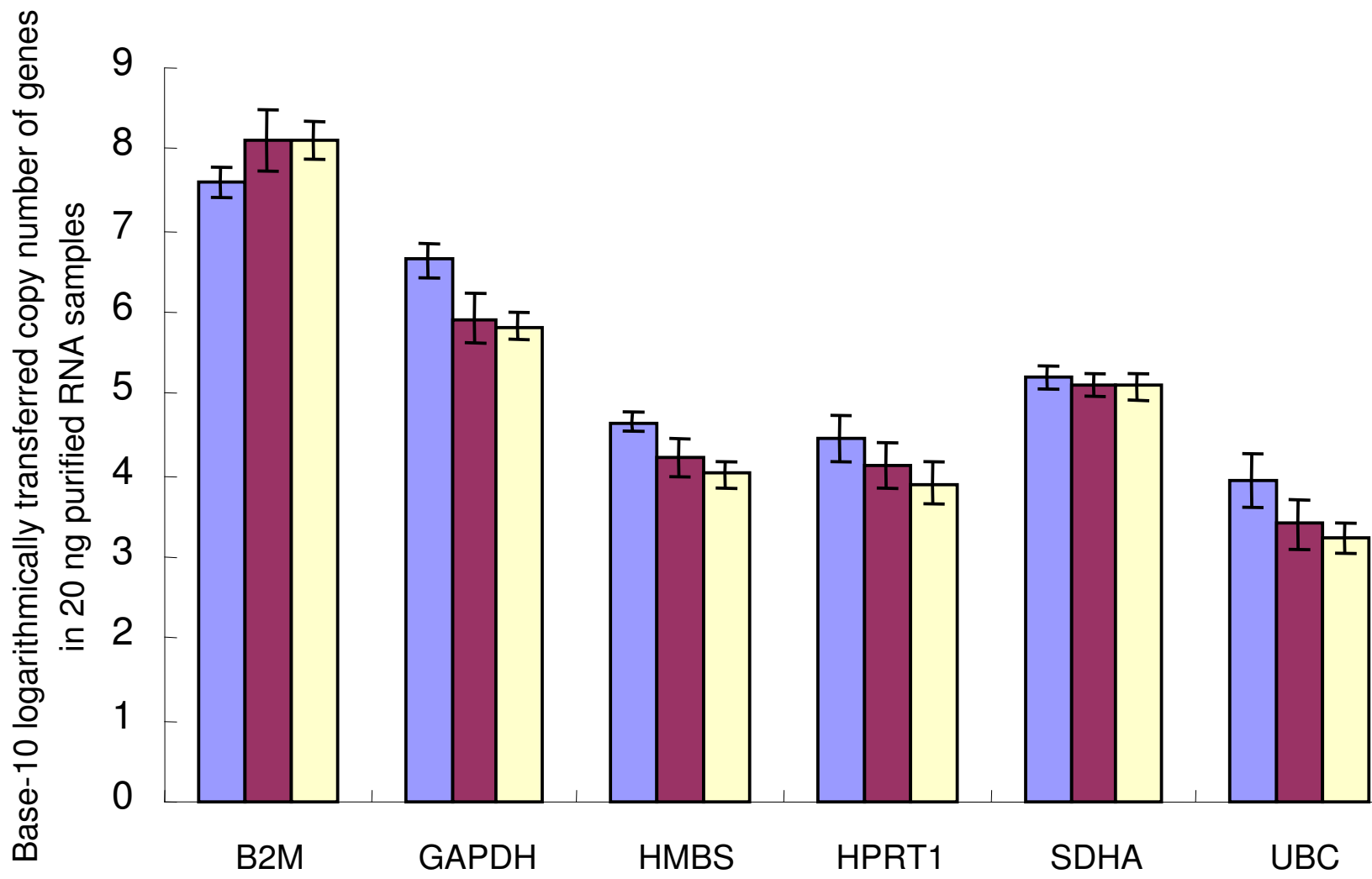

cell lines $\square$ liver cancer tissues $\square$ non-tumoral tissues

Figure 3

Expression levels of 6 putative reference genes in different sample panels. Average copy number of each gene in 20 $\mathrm{ng}$ of purified RNA samples was transferred on a base-I0 logarithmic scale. An approximately 10,000-fold expression difference is apparent between the most abundant (B2M) and the rarest (UBC) transcript.

Table 4: Reference genes ranked in order of increasing expression stability in paired liver tissues*.

\begin{tabular}{|c|c|c|c|c|c|c|c|}
\hline \multicolumn{4}{|c|}{ paired tissues $A(n=22)$} & \multicolumn{4}{|c|}{ Paired tissues B $(n=32)$} \\
\hline geNorm & $M$ value & NormFinder & Stability value & geNorm & $M$ value & NormFinder & Stability value \\
\hline$B 2 M$ & 0.799 & $B 2 M$ & 0.149 & $B 2 M$ & 0.826 & $U B C$ & 0.207 \\
\hline HPRTI & 0.658 & HPRTI & 0.105 & HPRTI & 0.816 & SDHA & 0.203 \\
\hline$U B C$ & 0.654 & $U B C$ & 0.101 & $U B C$ & 0.771 & HPRTI & 0.164 \\
\hline SDHA & 0.598 & GAPDH & 0.068 & SDHA & 0.711 & $B 2 M$ & 0.159 \\
\hline GAPDH & 0.566 & SDHA & 0.052 & GAPDH & 0.692 & GAPDH & 0.145 \\
\hline HMBS & 0.514 & HMBS & 0.021 & HMBS & 0.629 & HMBS & 0.084 \\
\hline HMBS-GAPDH & 0.417 & HMBS-SDHA & 0.033 & HMBS-SDHA & 0.489 & HMBS-B2M & 0.079 \\
\hline
\end{tabular}

Paired tissues A: paired tumoral and non-tumoral tissues left after the exclusion of RNA samples with relatively high amount of inhibitors; Paired tissues B: all paired tissues. *Increases from top to bottom. 
Table 5: Reference genes ranked in order of increasing expression stability in tumoral tissues and cell lines*.

\begin{tabular}{|c|c|c|c|c|c|c|c|}
\hline \multicolumn{4}{|c|}{ Tumoral tissues } & \multicolumn{4}{|c|}{ Cell lines } \\
\hline geNorm & $M$ value & NormFinder & Stability value & geNorm & $M$ value & NormFinder & Stability value \\
\hline$B 2 M$ & 1.091 & $B 2 M$ & 0.661 & $U B C$ & 1.277 & $U B C$ & 0.791 \\
\hline$S D H A$ & 0.953 & SDHA & 0.517 & HPRTI & 1.112 & HPRTI & 0.652 \\
\hline HPRTI & 0.863 & HPRTI & 0.448 & GAPDH & 1.002 & GAPDH & 0.457 \\
\hline$U B C$ & 0.837 & $U B C$ & 0.393 & SDHA & 0.921 & SDHA & 0.408 \\
\hline GAPDH & 0.763 & GAPDH & 0.294 & $B 2 M$ & 0.887 & $B 2 M$ & 0.321 \\
\hline HMBS & 0.676 & HMBS & 0.060 & HMBS & 0.857 & HMBS & 0.272 \\
\hline HMBS-GAPDH & $0.46 I$ & - & - & HMBS-SDHA & 0.620 & - & - \\
\hline
\end{tabular}

* Increases from top to bottom

It is worth noting that clonal variation that has been described to occur in HCC [34], might induce bias in gene expression studies. While genomic heterogeneity may interfere with gene expression studies aimed at identifying a malignant genotype, this phenomenon should be of less importance for the selection of reference genes which are constitutively expressed and are generally involved in basic functions needed for the sustenance of the cell.

As shown in Table 6, major differences were evident in the ranking order and gene stability of the candidate genes in paired tissues compared to a similar study previously published by Kim et al. [35]. UBC was ranked as the most sta-

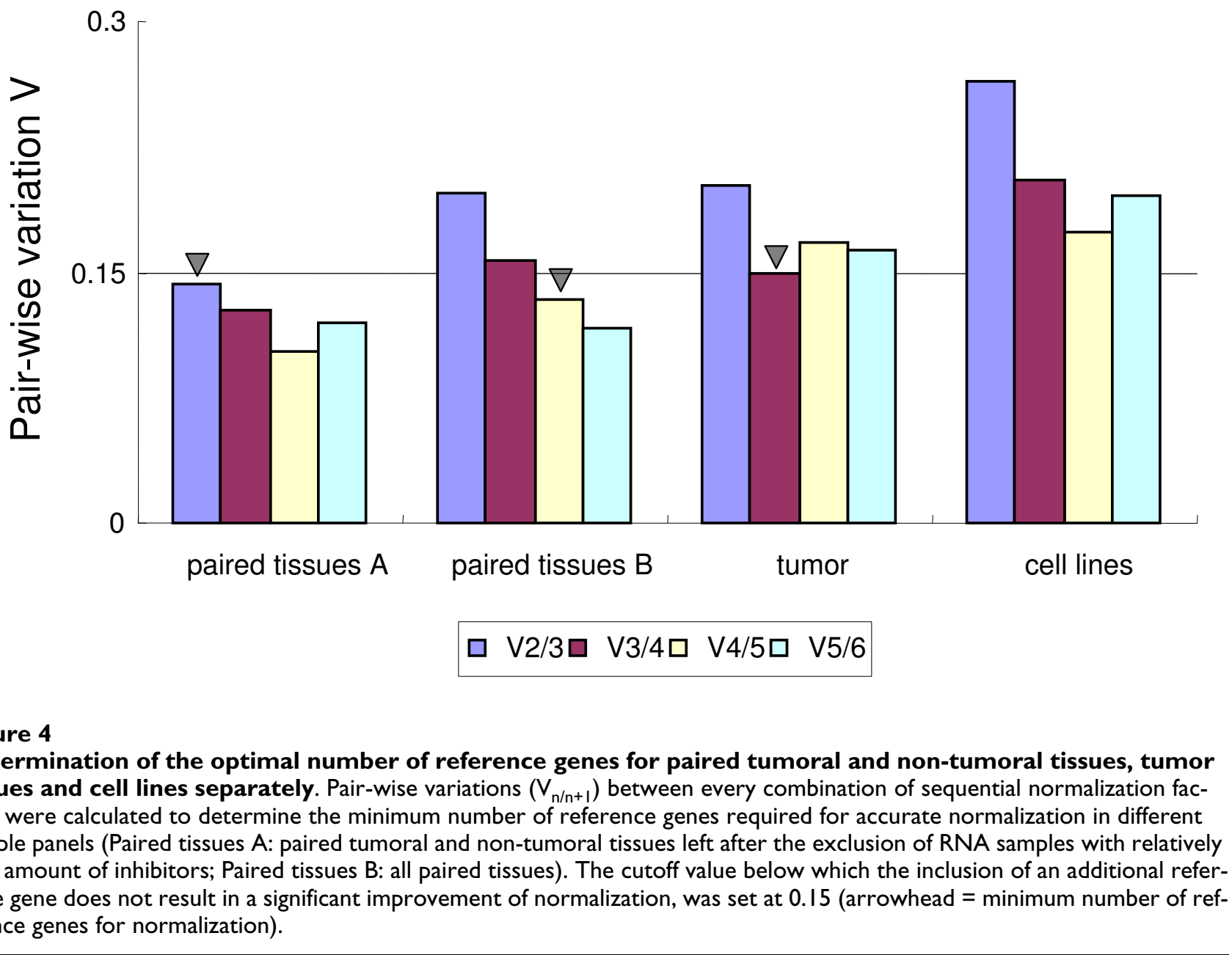


Table 6: Reference genes ranked in order of increasing expression stability in the current study and the study of Kim et al. [35].

\begin{tabular}{ccccc}
\hline & \multicolumn{2}{c}{ Current study* } & \multicolumn{2}{c}{ Study of Kim et al. } \\
\cline { 2 - 5 } Rank & Genes & M value & Genes & M value \\
\hline $\mathbf{6}$ & B2M & 0.799 & B2M & 2.60 \\
$\mathbf{5}$ & $H P R T I$ & 0.658 & RPLI3A & 2.50 \\
$\mathbf{4}$ & UBC & 0.654 & GAPDH & 2.43 \\
$\mathbf{3}$ & SDHA & 0.598 & HPRTI & 2.26 \\
$\mathbf{2}$ & GAPDH & 0.566 & HMBS & 2.18 \\
$\mathbf{I}$ & HMBS & 0.514 & UBC & 2.17 \\
& HMBS-GAPDH & 0.417 & UBC-HMBS & 1.02 \\
\hline
\end{tabular}

* Data shown are from the sample panel of combined tumoral and non-tumoral tissues

bly expressed gene in the previous study, but was ranked as the fourth using both geNorm and NormFinder in our study. In addition, the $\mathrm{M}$ values for the candidate reference genes were much lower in our study, thus indicating a high reliability of the data. The notably higher stability of putative reference genes in our study may be due to the following: (i) matching tumoral tissue with the nontumoral tissue from the same patient may have reduced the noise deriving from diversity of gene expression among individuals; (ii) the integrity of RNA was assessed and controlled using Agilent Bioanalyzer; (iii) qRT-PCR inhibitor was maintained at an acceptable level in all standards and samples; and (iv) qRT-PCR efficiency was determined for each primer pair.

\section{Conclusion}

Of six genes studied, HMBS was found to be the single best reference gene for expression studies in HCC. The appropriate choice of combination of more than one reference gene to improve qRT-PCR accuracy depends on the kind of liver tissues or cells under investigation. Quantitative assessment and control of qRT-PCR inhibitors using an external RNA control can reduce the variation of qRTPCR assay and facilitate the evaluation of gene stability. Our results may facilitate the choice of reference genes for expression studies in HCC.

\section{Abbreviations}

qRT-PCR: quantitative reverse transcription polymerase chain reaction; HCC: hepatocellular carcinoma; $\mathrm{B} 2 \mathrm{M}$ : beta-2-microglobulin; GAPDH: glyceraldehyde-3-phosphate dehydrogenase; HMBS: hydroxymethylbilane synthase; HPRT1: hypoxanthine guanine phosphoribosyl transferase 1; SDHA: succinate dehydrogenase flavoprotein subunit A; UBC: ubiquitin C; OD: optical density; RIN: RNA integrity number; $\mathrm{Ct}$ : threshold cycle; $\mathrm{M}$ : gene stability value; NF: normalization factor; HCV: hepatitis C virus; $\Delta$ Ct: delta threshold cycle.

\section{Competing interests}

The authors declare that they have no competing interests.

\section{Authors' contributions}

VRC and QS designed, performed, and analyzed the research and wrote the paper. GCS and AR participated in the data analysis. GG and SB conceived the study, participated in its design and helped to draft the manuscript. All authors read and approved the final manuscript.

\section{Additional material}

\section{Additional file 1}

Supplementary table 1-5. Supplementary table 1-5 (doc format) indicate the sequences of primers for cloning and the length of amplicons, background of cell lines, background of liver tissue samples, copy number of the standards, and RNA quality.

Click here for file

[http://www.biomedcentral.com/content/supplementary/14712407-8-350-S1.doc]

\section{Additional file 2}

Melting curve analysis obtained for the $\mathrm{B} 2 \mathrm{M}$ gene. Melt curve peak chart (rtf format) collected using the Bio-Rad iQ5 Software 2.0 (Bio$\mathrm{Rad}$ ) during calibration experiments of the selected primer pair for the $\mathrm{B} 2 \mathrm{M}$ gene on an $\mathrm{iQ}^{\mathrm{TM}} 5 \mathrm{Multicolor} \mathrm{Real-Time} \mathrm{PCR} \mathrm{Detection} \mathrm{System} \mathrm{(Bio-}$ Rad). RFU: relative fluorescence units; T: temperature.

Click here for file

[http://www.biomedcentral.com/content/supplementary/14712407-8-350-S2.rtf]

\section{Additional file 3}

Melting curve analysis obtained for the GAPDH gene. Melt curve peak chart (rtf format) collected using the Bio-Rad iQ5 Software 2.0 (BioRad) during calibration experiments of the selected primer pair for the GAPDH gene on an $i Q^{\mathrm{TM}} 5$ Multicolor Real-Time PCR Detection System (Bio-Rad). RFU: relative fluorescence units; T: temperature. Click here for file [http://www.biomedcentral.com/content/supplementary/14712407-8-350-S3.rtf]

\section{Additional file 4}

Melting curve analysis obtained for the HMBS gene. Melt curve peak chart (rtf format) collected using the Bio-Rad iQ5 Software 2.0 (Bio$\mathrm{Rad}$ ) during calibration experiments of the selected primer pair for the HMBS gene on an $i^{\mathrm{TM}} 5$ Multicolor Real-Time PCR Detection System (Bio-Rad). RFU: relative fluorescence units; T: temperature.

Click here for file

[http://www.biomedcentral.com/content/supplementary/14712407-8-350-S4.rtf]

\section{Additional file 5}

Melting curve analysis obtained for the HPRT1 gene. Melt curve peak chart (rtf format) collected using the Bio-Rad iQ5 Software 2.0 (BioRad) during calibration experiments of the selected primer pair for the HPRT1 gene on an $i^{\mathrm{TM}} 5$ Multicolor Real-Time PCR Detection System (Bio-Rad). RFU: relative fluorescence units; T: temperature.

Click here for file

[http://www.biomedcentral.com/content/supplementary/14712407-8-350-S5.rtf] 


\section{Additional file 6}

Melting curve analysis obtained for the SDHA gene. Melt curve peak chart (rtf format) collected using the Bio-Rad iQ5 Software 2.0 (BioRad) during calibration experiments of the selected primer pair for the SDHA gene on an $i^{\mathrm{TM}} 5$ Multicolor Real-Time PCR Detection System (Bio-Rad). RFU: relative fluorescence units; T: temperature.

Click here for file

[http://www.biomedcentral.com/content/supplementary/14712407-8-350-S6.rtf]

\section{Additional file 7}

Melting curve analysis obtained for the UBC gene. Melt curve peak chart (rtf format) collected using Bio-Rad iQ5 Software 2.0 (Bio-Rad) during calibration experiments of the selected primer pair for the UBC gene on an ${ }^{2} Q^{\mathrm{TM}} 5$ Multicolor Real-Time PCR Detection System (Bio-Rad) $R F U$ : relative fluorescence units; T: temperature.

Click here for file

[http://www.biomedcentral.com/content/supplementary/14712407-8-350-S7.rtf]

\section{Additional file 8}

PCR amplification chart for B2M and GAPDH genes in two samples containing genomic DNA. PCR amplification chart (rtf format) collected using Bio-Rad iQ5 Software 2.0 (Bio-Rad) during calibration experiments of the selected primer pair for the $\mathrm{B} 2 \mathrm{M}$ and GAPDH genes on an $i^{\mathrm{Tm}} 5$ Multicolor Real-Time PCR Detection System (Bio-Rad) in two samples still contained genomic DNA as shown in previous RT negative controls. The Ct values of RT negative reactions were 16.37-17.94 lower than that of RT positive control. A: RT positive reaction. B: RT negative reaction for the same samples. RFU: relative fluorescence units; $T$ : temperature.

Click here for file

[http://www.biomedcentral.com/content/supplementary/14712407-8-350-S8.rtf]

\section{Acknowledgements}

The authors thank Dr. Brigitte Pützer for kindly providing the $\mathrm{HuH} 7$ cell line, Dr. Frank Simon for helpful suggestions, and Anne Achterfeld for technical assistance. This study was funded in part by 107-057 I0/IFORES program (VRC) and 107-05470/IFORES program (SB).

\section{References}

I. Vandesompele J, De PK, Pattyn F, Poppe B, Van RN, De PA, et al: Accurate normalization of real-time quantitative RT-PCR data by geometric averaging of multiple internal control genes. Genome Biol 2002, 3:RESEARCH0034.

2. Ginzinger DG: Gene quantification using real-time quantitative PCR: an emerging technology hits the mainstream. Exp Hematol 2002, 30:503-512.

3. Huggett J, Dheda K, Bustin S, Zumla A: Real-time RT-PCR normalisation; strategies and considerations. Genes Immun 2005, 6:279-284

4. Dheda K, Huggett JF, Bustin SA, Johnson MA, Rook G, Zumla A: Validation of housekeeping genes for normalizing RNA expression in real-time PCR. BioTechniques 2004, 37: I I 2-1 I 9.

5. Dheda K, Huggett JF, Chang JS, Kim LU, Bustin SA, Johnson MA, et al.: The implications of using an inappropriate reference gene for real-time reverse transcription PCR data normalization. Anal Biochem 2005, 344:|4I-I43.

6. Valenti MT, Bertoldo F, Dalle CL, Azzarello G, Zenari S, Zanatta M, et al: The effect of bisphosphonates on gene expression: GAPDH as a housekeeping or a new target gene? BMC Cancer 2006, 6:49.
7. Selvey S, Thompson EW, Matthaei K, Lea RA, Irving MG, Griffiths LR: Beta-actin - an unsuitable internal control for RT-PCR. Mol Cell Probes 200I, I5:307-3II.

8. Arukwe A: Toxicological housekeeping genes: do they really keep the house? Environ Sci Technol 2006, 40:7944-7949.

9. Tricarico C, Pinzani P, Bianchi S, Paglierani M, Distante V, Pazzagli M, et al.: Quantitative real-time reverse transcription polymerase chain reaction: normalization to rRNA or single housekeeping genes is inappropriate for human tissue biopsies. Anal Biochem 2002, 309:293-300.

10. Thellin O, Zorzi W, Lakaye B, De BB, Coumans B, Hennen G, et al: Housekeeping genes as internal standards: use and limits. Biotechnol 1999, 75:291-295.

II. Tanic N, Perovic M, Mladenovic A, Ruzdijic S, Kanazir S: Effects of aging, dietary restriction and glucocorticoid treatment on housekeeping gene expression in rat cortex and hippocampus-evaluation by real time RT-PCR. J Mol Neurosci 2007, 32:38-46.

12. Waxman S, Wurmbach E: De-regulation of common housekeeping genes in hepatocellular carcinoma. BMC Genomics 2007, 8:243

13. Parkin DM, Pisani P, Ferlay J: Global cancer statistics. CA Cancer J Clin 1999, 49:33-64

14. Fang H, Tong W, Perkins R, Shi L, Hong H, Cao X, et al.: Bioinformatics approaches for cross-species liver cancer analysis based on microarray gene expression profiling. BMC Bioinformatics 2005, 6(Suppl 2):S6.

15. Segal E, Sirlin CB, Ooi C, Adler AS, Gollub J, Chen X, et al.: Decoding global gene expression programs in liver cancer by noninvasive imaging. Nat Biotechnol 2007, 25:675-680.

16. Li B, Perabekam S, Liu G, Yin M, Song S, Larson A: Experimental and bioinformatics comparison of gene expression between $T$ cells from TIL of liver cancer and T cells from UniGene. J Gastroenterol 2002, 37:275-282.

17. Wakabayashi K, Saito H, Kaneko F, Nakamoto N, Tada S, Hibi T: Gene expression associated with the decrease in malignant phenotype of human liver cancer cells following stimulation with a histone deacetylase inhibitor. Int J Oncol 2005, 26:233-239.

18. Kim JW, Wang XW: Gene expression profiling of preneoplastic liver disease and liver cancer: a new era for improved early detection and treatment of these deadly diseases? Carcinogenesis 2003, 24:363-369.

19. Choi JK, Choi JY, Kim DG, Choi DW, Kim BY, Lee KH, et al:: Integrative analysis of multiple gene expression profiles applied to liver cancer study. FEBS Lett 2004, 565:93-100.

20. Chenivesse $X$, Franco D, Brechot C: MDRI (multidrug resistance) gene expression in human primary liver cancer and cirrhosis. J Hepatol 1993, 18:168-172.

21. Pattyn F, Robbrecht P, De PA, Speleman F, Vandesompele J: RTPrimerDB: the real-time PCR primer and probe database, major update 2006. Nucleic Acids Res 2006, 34:D684-D688.

22. Schroeder A, Mueller O, Stocker S, Salowsky R, Leiber M, Gassmann $M$, et al: The RIN: an RNA integrity number for assigning integrity values to RNA measurements. BMC Mol Biol 2006, 7:3.

23. Arezi B, Salehi M: High-quality external RNA control detects inhibitors in RNA samples. Strategies 2007, 19:52-53.

24. Warner JR: The economics of ribosome biosynthesis in yeast. Trends Biochem Sci 1999, 24:437-440.

25. Solanas M, Moral R, Escrich E: Unsuitability of using ribosomal RNA as loading control for Northern blot analyses related to the imbalance between messenger and ribosomal RNA content in rat mammary tumors. Anal Biochem 200I, 288:99-102.

26. Spanakis E: Problems related to the interpretation of autoradiographic data on gene expression using common constitutive transcripts as controls. Nucleic Acids Res 1993, 21:3809-38I9.

27. Johnson ML, Redmer DA, Reynolds LP: Quantification of lane-tolane loading of poly(A) RNA using a biotinylated oligo(dT) probe and chemiluminescent detection. BioTechniques 1995 , 19:7I2-7I5.

28. Luebbehusen $\mathrm{H}$ : The Significance of the 260/230 Ratio in Determining Nucleic Acid Purity. 2004 [http://www.bcm.edu/ mcfweb/?PMID=3100].

29. Fleige S, Pfaffl MW: RNA integrity and the effect on the realtime qRT-PCR performance. Mol Aspects Med 2006, 27: I26-139. 
30. Kim CH, Khan M, Morin DE, Hurley WL, Tripathy DN, Kehrli M Jr, et al:: Optimization of the PCR for detection of Staphylococcus aureus nuc gene in bovine milk. J Dairy Sci 200I, 84:74-83.

31. Bessetti ]: An Introduction to PCR Inhibitors. Profiles in DNA 2007:9-10.

32. Freeman WM, Walker SJ, Vrana KE: Quantitative RT-PCR: pitfalls and potential. BioTechniques 1999, 26: I I2-II5.

33. Andersen CL, Jensen JL, Orntoft TF: Normalization of real-time quantitative reverse transcription-PCR data: a model-based variance estimation approach to identify genes suited for normalization, applied to bladder and colon cancer data sets. Cancer Res 2004, 64:5245-5250.

34. Thorgeirsson SS, Grisham JW: Molecular pathogenesis of human hepatocellular carcinoma. Nat Genet 2002, 31 :339-346.

35. Kim S, Kim T: Selection of optimal internal controls for gene expression profiling of liver disease. BioTechniques 2003, 35:456-460.

\section{Pre-publication history}

The pre-publication history for this paper can be accessed here:

http://www.biomedcentral.com/1471-2407/8/350/pre

pub

Publish with Biomed Central and every scientist can read your work free of charge

"BioMed Central will be the most significant development for disseminating the results of biomedical research in our lifetime. "

Sir Paul Nurse, Cancer Research UK

Your research papers will be:

- available free of charge to the entire biomedical community

- peer reviewed and published immediately upon acceptance

- cited in PubMed and archived on PubMed Central

- yours - you keep the copyright

Submit your manuscript here:

http://www.biomedcentral.com/info/publishing_adv.asp
BioMedcentral 